\title{
Convergence Rates for Finite Element Approximations of Stochastic Partial Differential Equations
}

\author{
Fred Espen Benth ${ }^{1} \&$ Jon Gjerde ${ }^{2}$
}

\begin{abstract}
We study convergence rates for generalized random variables. These results will be applied to study a stochastic finite element approach to stochastic partial differential equations.
\end{abstract}

Mathematics Subject Classifications (1991). 60G20, 60H15, 60H30.

Keywords: Stochastic partial differential equations, chaos expansions, finite element method.

\section{$\S 1$ Introduction}

In recent years there has been a growing interest in stochastic partial differential equations as models of physical systems perturbed by noise. Øksendal et al. [HLØUZ] proposed a stochastic version of the pressure equation for fluid flow in a porous medium. In Walsh, [W] we find a stochastic partial differential equation describing the behavior of neurons. To solve the nonlinear filtering problem, the well-known Zakai-equation appears as the model for the (unnormalized) density of the solution (see e.g. Zakai [Z]).

There exists an extensive literature on how to solve deterministic partial differential equations numerically. For stochastic problems of the same nature, the theory seems to be not so well developed. However, there exists some references. We want to mention the paper of $\mathrm{Hu},[\mathrm{Hu}]$ which considers the pressure equation model of Øksendal et al. [HLØUZ]. He treats this stochastic equation by approximating derivatives with finite differences. However, there is a problem of

\footnotetext{
${ }^{1}$ Norwegian Computing Center, P. O. Box 114 Blindern, N-0314 Oslo, Norway, E-mail: Fred.Espen.Benth@nr.no

${ }^{2}$ Department of Mathematics, University of Oslo, P. O. Box 1053 Blindern, N-0316 Oslo, Norway,

E-mail: Gjerde@math.uio.no
} 
calculating a combination of random variables called the Wick product. Germani and Piccioni, [GP] show how to solve the above mentioned Zakai- equation using finite element methods. A thorough treatment of numerical methods for ordinary stochastic differential equations can be found in the monograph by Kloeden and Platen [KP].

The main objective of this paper is to give a general framework to solve elliptic stochastic partial differential equations based on the finite element method. The class of problems we have in mind is on the form

$$
\begin{cases}\nabla(k(x, \omega) \cdot \nabla u(x, \omega))=-f(x, \omega), & (x, \omega) \in D \times \Omega \\ u(x, \omega)=g(x, \omega), & (x, \omega) \in \partial D \times \Omega\end{cases}
$$

where $D$ is a bounded domain in $\mathbb{R}^{2}$ and $\Omega$ is a probability space. $f$ and $g$ are $\mathbb{R}$-valued functions on $D \times \Omega$ and $k: D \times \Omega \rightarrow \mathbb{R}^{2 \times 2}$. The weak formulation of such problems will be considered to provide numerical schemes. In Våge, [V] elliptic problems have been studied in the framework of Wick Calculus. We remark that our approach fits every elliptic problem that can be rewritten in a variational form. See section 5 for a precise statement. We refer to [C] for the finite element method for $\mathbb{R}^{n}$.

Our approach is a direct application of the Ritz-Galerkin method put in a new setting. Since we deal with stochastic equations, a new independent variable $\omega$ comes in addition to the usual space variable $x . \omega$ is an event in the probability space $\Omega$. Due to this extra variable, new Hilbert spaces are called for to fit the framework of the Ritz-Galerkin method. Such spaces are introduced in this paper as the topological sum of a classical Sobolev space and a Hilbert space taking the stochastic regularity into account. Convergence rates are calculated. In order to achieve numerically treatable problems, we need an explicit basis for the Hilbert spaces expressing the stochastic regularity of a solution. This can found using the standard spaces found in the White Noise Analysis. These spaces are known under the names Hida and Kondratiev distribution spaces. As indicated by the names, they contain generalized random variables, i.e. random variables that are not necessarily square integrable. For more information, we refer to the works of Hida et al. [HKPS] and Kondratiev et al. [KLS]. We note that the Meyer-Watanabe distribution space is included in the Hida distribution space.

We want our numerical approach to include elliptic stochastic partial differential equations having singular solutions. The reason for this is that such problems in general have solutions that do not have finite variance. In fact, they sometimes even fail to be integrable. Examples of such a behavior can be found in [W] and [HLØUZ]. It is hence natural to consider Hilbert spaces containing generalized random variables. However, elliptic stochastic problems having smooth solutions (in $\omega)$ are of course included! Unfortunately, our methods seems to get very computer extensive. We will in a future paper test out the methods on a computer.

The numerical approach in this paper are taken from the book of Hackbusch [H]. We also base our presentation on the White Noise Analysis. The interested reader is advised to look into Hida et al. [HKPS], Øksendal et al. [HØUZ] and Kondratiev et al. [KLS].

The paper proceeds as follows: In section 2 we introduce some notation and the Sobolev spaces 
that will be used. A convergence rate estimate for a finite dimensional approximation of generalized random variables is calculated in section 3. We follow up with the Sobolev analogue of such a result in the next section. Finally, in section 5 we apply our results to elliptic stochastic problems on a variational form. In an appendix we discuss some computional aspects.

\section{$\S 2$ Mathematical Preliminaries}

We will in the following fix the parameter dimension $d \in N$. Let $\mathcal{S}=\mathcal{S}\left(\mathbb{R}^{d}\right)$ be the Schwartz space of rapidly decreasing smooth functions on $\mathbb{R}^{\mathrm{d}}$. The dual $\mathcal{S}^{\prime}=\mathcal{S}^{\prime}\left(\mathbb{R}^{\mathrm{d}}\right)$, equipped with the weak-star topology, is the space of tempered distributions. By the Bochner-Minlos theorem there exists a probability measure $\mu$ on the Borel subsets $\mathcal{B}$ of $\mathcal{S}^{\prime}$ defined by the characteristic functional

$$
\int_{\mathcal{S}^{\prime}} e^{i<\omega, \phi>} d \mu=e^{-\frac{1}{2}|\phi|_{2}^{2}} ; \forall \phi \in \mathcal{S}
$$

where $|\cdot|_{2}$ is the norm on $L^{2}\left(\mathbb{R}^{d}\right)$.

Let now $\mathcal{J}:=\left(\mathbf{N}_{0}^{\mathbb{N}}\right)_{c}$, i.e. the set of all sequences $\alpha=\left(\alpha_{1}, \alpha_{2}, \cdots\right)$ with elements $\alpha_{i} \in N_{0}$ and only finitely many $\alpha_{i} \neq 0$.

An orthogonal basis for $L^{2}(\mu)$ is given by $\left\{H_{\alpha}\right\}_{\alpha \in \mathcal{J}}$ where $H_{\alpha}(\omega)$ is defined as

$$
H_{\alpha}(\omega):=\prod_{i=1}^{\ell(\alpha)} h_{\alpha_{i}}\left(\left\langle\omega, \xi_{i}\right\rangle\right)
$$

$\ell(\alpha)$ is the length of the multi-index $\alpha$ and $\left\{\xi_{i}\right\}_{i=1}^{\infty}$ is any orthonormal basis consisting of orthonormal tensor products of the Hermite functions in $L^{2}(\mathbb{R}) . h_{n}$ is the Hermite polynomial of order $n$.

Let $V$ be any real Hilbert space. Then we define the Hilbert spaces $(\mathcal{S})^{\rho, k, V}(\rho \in[-1,1], k \in \mathbb{R})$ as the set of formal sums

$$
F=\sum_{\alpha \in \mathcal{J}} f_{\alpha} H_{\alpha} ; f_{\alpha} \in V(\forall \alpha \in \mathcal{J})
$$

with finite norm $\|F\|_{\rho, k, V}$ induced from the inner product

$$
(F, G)_{\rho, k, V}:=\sum_{\alpha \in \mathcal{J}}\left(f_{\alpha}, g_{\alpha}\right)_{V}(\alpha !)^{1+\rho}(2 N)^{k \alpha}
$$

where

$$
(2 \mathbf{N})^{k \alpha}:=\prod_{j}(2 j)^{\alpha_{j}}
$$

Note that $(\rho>0)(\mathcal{S})^{-\rho,-k, V}$ is the dual space of $(\mathcal{S})^{\rho, k, V}$, The dual action is defined by

$$
\langle F, f\rangle:=\sum_{\alpha \in \mathcal{J}}\left(F_{\alpha}, f_{\alpha}\right)_{v} \alpha !
$$


where $f \in(\mathcal{S})^{\rho, k, V}$ and $F \in(\mathcal{S})^{-\rho,-k, V}$.

When $V=H^{m}$ or $V=H_{0}^{m}$, we will use the notation $(\mathcal{S})^{\rho, k, m}$ and $(\mathcal{S})_{0}^{\rho, k, m}$ respectively. In the case when $V=\mathbb{R}$ we will use the notation $(\mathcal{S})^{\rho, k}$ and also define the familiar spaces $(\mathcal{S})^{\rho}:=$ $\cap_{k \geq 0}(\mathcal{S})^{\rho, k, V}$ and $(\mathcal{S})^{-\rho}:=\cup_{k \geq 0}(\mathcal{S})^{-\rho,-k, V} ; \rho \in[0,1]$.

More information can be found in [HØUZ] and [HKPS].

\section{$\S 3$ Approximation of generalized random variables}

Introduce the set of multi-indices

$$
\mathcal{A}_{n, k}=\left\{\alpha \in \mathbf{N}_{0}^{k} \mid \alpha_{k} \neq 0, \alpha_{1}+\ldots+\alpha_{k}=n\right\}
$$

where $n, k \in N$. Obviously, $\alpha \in \mathcal{A}_{n, k}$ has length $k$.

Let $\Phi \in(\mathcal{S})^{-\rho,-q, V}$ be given as

$$
\Phi=\sum_{\alpha \in \mathcal{J}} c_{\alpha} H_{\alpha}
$$

and for $\mathrm{N}, \mathrm{K} \in \mathrm{N}$ define the finite dimensional approximation

$$
\Phi^{\mathrm{N}, \mathrm{K}}:=c_{0}+\sum_{n=1}^{\mathrm{N}} \sum_{\mathrm{k}=1}^{\mathrm{K}} \sum_{\alpha \in \mathcal{A}_{n, \mathrm{~K}}} \mathrm{c}_{\alpha} \mathrm{H}_{\alpha}
$$

We have the following result on the convergence:

THEOREM 3.1 Let $p \in \mathbb{R}_{+}$be given and assume that $r:=p-q>r^{*}$, where $r^{*}$ solves

$$
\frac{r^{*}}{2^{*}\left(r^{*}-1\right)}=1
$$

Then for any $\psi \in(\mathcal{S})^{p, q, V}$

$$
\left|\left\langle\Phi-\Phi^{N, K}, \psi\right\rangle\right| \leq\|\Phi\|_{-\rho,-q, V} \cdot\|\psi\|_{\rho, p, V} \cdot \sqrt{C_{1}(r) \cdot K^{1-r}+C_{2}(r) \cdot\left(\frac{r}{2^{r}(r-1)}\right)^{N+1}}
$$

where

$$
C_{1}(r)=\frac{1}{2^{r}(r-1)-r} \text { and } C_{2}(r)=2^{r}(r-1) C_{1}(r)
$$

Note that $r^{*}$ is approximately 1.53 .

\section{PROOF:}


Let

$$
c_{n, k}=\sum_{\alpha \in \mathcal{A}_{n, k}} c_{\alpha} H_{\alpha}
$$

We see that

$$
\begin{aligned}
\Phi-\Phi^{N, K} & =\sum_{n=1}^{\infty} \sum_{k=1}^{\infty} c_{n, k}-\sum_{n=1}^{N} \sum_{k=1}^{K} c_{n, k} \\
& =\sum_{n=1}^{N} \sum_{k=1}^{\infty} c_{n, k}+\sum_{n=N+1}^{\infty} \sum_{k=1}^{\infty} c_{n, k}-\sum_{n=1}^{N} \sum_{k=1}^{K} c_{n, k} \\
& =\sum_{n=1}^{N} \sum_{k=k+1}^{\infty} c_{n, k}+\sum_{n=N+1}^{\infty} \sum_{k=1}^{\infty} c_{n, k}
\end{aligned}
$$

These two sums are the chaos expansion of $\Phi-\Phi^{N, K}$. By the definition of the $\|\cdot\|_{-p,-p, v}-$ norm and orthogonality of $\mathrm{H}_{\alpha}$ we have

$$
\begin{aligned}
& \left\|\Phi-\Phi^{N, K}\right\|_{-p,-p, V}^{2}=\sum_{n=1}^{N} \sum_{k=K+1}^{\infty} \sum_{\alpha \in \mathcal{A}_{n, k}}\left\|c_{\alpha}\right\|_{v}^{2}(\alpha !)^{1-\rho}(2 N)^{\alpha p} \\
& +\sum_{n=N+1}^{\infty} \sum_{k=1}^{\infty} \sum_{\alpha \in \mathcal{A}_{n, k}}\left\|c_{\alpha}\right\|_{V}^{2}(\alpha !)^{1-\rho}(2 N)^{\alpha p} \\
& =\sum_{n=1}^{N} \sum_{k=K+1}^{\infty} \sum_{\alpha \in \mathcal{A}_{n, k}}\left\|c_{\alpha}\right\|_{V}^{2}(\alpha !)^{1-\rho}(2 N)^{\alpha r}(2 N)^{\alpha q} \\
& +\sum_{n=N+1}^{\infty} \sum_{k=1}^{\infty} \sum_{\alpha \in \mathcal{A}_{n, k}}\left\|c_{\alpha}\right\|_{V}^{2}(\alpha !)^{1-\rho}(2 N)^{-2 \alpha \tau}(2 N)^{\alpha q} \\
& \leq\|\Phi\|_{-\rho,-q, V}^{2} \cdot\left(\sum_{n=1}^{N} \sum_{k=K+1}^{\infty} \sum_{\alpha \in \mathcal{A}_{n, k}}(2 N)^{-\alpha \mathrm{r}}+\sum_{n=N+1}^{\infty} \sum_{k=1}^{\infty} \sum_{\alpha \in \mathcal{A}_{n, k}}(2 N)^{-\alpha r}\right)
\end{aligned}
$$

In the last inequality we have used the obvious relation

$$
\left\|c_{\alpha}\right\|_{V}^{2}(\alpha !)^{1-\rho}(2 \mathbf{N})^{-\alpha q} \leq\|\Phi\|_{-\rho,-q, V}^{2} \quad(\forall \alpha \in \mathcal{J})
$$

To estimate the two sums, we rewrite the multi-indices in a convenient way: Every $\alpha \in \mathcal{A}_{\mathrm{n}, \mathrm{k}}$ can be written as

$$
\alpha=\epsilon_{i_{1} i_{2} \cdots i_{n}}
$$

where

$$
\epsilon_{i_{1} i_{2} \ldots i_{n}}=(0, \ldots, 0,1,0, \ldots, 0,1,0, \ldots, 0,1)
$$

The 1's appear on the coordinates $i_{1}, i_{2}$ etc. Moreover, we use the convention that if $i_{2}=i_{3}$, then we get the value 2 in coordinate $i_{2}$. Equivalently, if $i_{4}=i_{5}=i_{6}$ we get 3 in coordinate 
$i_{4}$. Hence, the number of repetitions of a coordinate $i_{j}$ yields the value in that coordinate. For example is $\epsilon_{3333}=(0,0,4)$. Note that $\alpha \in \mathcal{A}_{n, k}$ implies $i_{n}=k$. Moreover, the $i_{j}$ 's are ordered like

$$
k=i_{n} \geq i_{n-1} \geq i_{n-2} \geq \ldots \geq i_{1} \geq 1
$$

With this notation,

$$
(2 N)^{\alpha r}=(2 N)^{\epsilon_{i_{1}} \ldots i_{n} r}=\prod_{j=1}^{n}\left(2 i_{j}\right)^{-r}=2^{-n r} \cdot i_{1}^{-r} i_{2}^{-r} \ldots i_{n}^{-r}
$$

Here, $i_{n}=k$. To make the argument below easy to follow, we reorder the $i_{j}$ 's:

$$
1 \leq i_{n} \leq \ldots \leq i_{2} \leq i_{1}=k
$$

Let for the moment $M$ be a natural number. We have

$$
\sum_{k=M}^{\infty} \sum_{\alpha \in \mathcal{A}_{n, k}}(2 \mathbf{N})^{-\alpha r}=2^{-n r} \sum_{i_{1}=M}^{\infty} i_{1}^{-r} \sum_{i_{2}=1}^{i_{1}} i_{2}^{-r} \ldots \sum_{i_{n}=1}^{i_{n-1}} i_{n}^{-r}
$$

In order to prove the theorem, we estimate the two sums

$$
\begin{aligned}
I_{N, K} & :=\sum_{n=1}^{N} 2^{-n r} \sum_{i_{1}=K+1}^{\infty} i_{1}^{-r} \sum_{i_{2}=1}^{i_{1}} i_{2}^{-r} \cdots \sum_{i_{n}=1}^{i_{n-1}} i_{n}^{-r} \\
J_{N} & :=\sum_{n=N+1}^{\infty} 2^{-n r} \sum_{i_{1}=1}^{\infty} i_{1}^{-r} \sum_{i_{2}=1}^{i_{1}} i_{2}^{-r} \cdots \sum_{i_{n}=1}^{i_{n-1}} i_{n}^{-r}
\end{aligned}
$$

The rest of the proof is standard estimations of these two sums:

We show that

$$
I_{N, K} \leq \frac{1}{2^{r}(r-1)-r} \cdot K^{1-r}
$$

where $r>r^{*}$ : First, a standard estimation technique using integration yields

$$
\begin{aligned}
\sum_{i_{n}=1}^{i_{n-1}} i_{n}^{-r} & \leq 1+\int_{1}^{i_{n-1}} x^{-r} d x \\
& =1+\frac{1}{r-1}\left(1-\left(\frac{1}{i_{n-1}}\right)^{r-1}\right)
\end{aligned}
$$

Because of our assumptions that $r-1>r-r^{*}>0$ and

$$
i_{n-1} \leq i_{n-2} \leq \ldots \leq i_{1}
$$

we have

$$
\left(1-\left(\frac{1}{i_{n-1}}\right)^{r-1}\right) \leq 1-\left(\frac{1}{i_{1}}\right)^{r-1}
$$


The rest of the sums are estimated the same way, ending up with the bound

$$
\begin{aligned}
\sum_{i_{2}=1}^{i_{1}} i_{2}^{-r} \cdots \sum_{i_{n}=1}^{i_{n-1}} i_{n}^{-2 r} & \leq\left(1+\frac{1}{r-1}\left(1-\left(\frac{1}{i_{1}}\right)^{r-1}\right)\right)^{n-1} \\
& =\left(\frac{1}{r-1}\right)^{n-1}\left(r-\left(\frac{1}{i_{1}}\right)^{r-1}\right)^{n-1} \\
& \leq\left(\frac{1}{r-1}\right)^{n-1} r^{n-1}
\end{aligned}
$$

and hence

$$
\begin{aligned}
\sum_{i_{1}=k+1}^{\infty}\left(\sum_{i_{2}=1}^{i_{2}} i_{2}^{-r} \cdots \sum_{i_{n}=1}^{i_{n-1}} i_{n}^{-r}\right) & \leq\left(\frac{1}{r-1}\right)^{n-1} \sum_{i=k+1}^{\infty} i^{-r} r^{n-1} \\
& =\left(\frac{r}{r-1}\right)^{n-1} \sum_{i=K+1}^{\infty} i^{-r}
\end{aligned}
$$

Since

$$
\sum_{i=K+1}^{\infty} i^{-r} \leq \int_{K}^{\infty} x^{-r} d x=\frac{K^{1-r}}{r-1}
$$

when $r>r^{*}>1$, we have the bound

$$
\sum_{i_{1}=K+1}^{\infty}\left(\sum_{i_{2}=1}^{i_{1}} i_{2}^{-r} \cdots \sum_{i_{n}=1}^{i_{n-1}} i_{n}^{-r}\right) \leq\left(\frac{r}{r-1}\right)^{n} \frac{K^{1-r}}{r}
$$

Finally, we obtain the bound

$$
\begin{aligned}
I_{N, K} & \leq \frac{K^{1-r}}{r} \sum_{\substack{n=1 \\
K^{1-r}}}^{N}\left(\frac{r}{2^{r}(r-1)}\right)^{n} \\
& \leq \frac{K^{r}(r-1)-r}{r^{r}(r)}
\end{aligned}
$$

whenever $r>r^{*}$. The constant $r^{*}$ solves

$$
\frac{r^{*}}{2^{r^{*}}\left(r^{*}-1\right)}=1
$$

which implies that

$$
\frac{r}{2^{r}(r-1)}<1
$$

for every $r>r^{*}$. Solving for $r^{*}$ numerically gives the estimate 1.53 . 
Finally we show that

$$
J_{N} \leq\left(\frac{2^{r}(r-1)}{2^{r}(r-1)-r}\right)\left(\frac{r}{2^{r}(r-1)}\right)^{N+1}
$$

Estimating as above,

$$
\sum_{i_{2}=1}^{i_{1}} i_{2}^{-r} \cdots \sum_{i_{n}=1}^{i_{n-1}} i_{n}^{-r} \leq\left(\frac{r}{r-1}\right)^{n-1}
$$

Furthermore,

$$
\begin{aligned}
\sum_{i_{1}=1}^{\infty} i_{1}^{-r} \sum_{i_{2}=1}^{i_{1}} i_{2}^{-r} \ldots \sum_{i_{n}=1}^{i_{n-1}} i_{n}^{-r} & \leq\left(\frac{r}{r-1}\right)^{n-1} \sum_{i_{1}=1}^{\infty} i_{1}^{-r} \\
& \leq\left(\frac{r}{r-1}\right)^{n-1}\left(1+\int_{1}^{\infty} x^{-r} d x\right) \\
& =\left(\frac{r}{r-1}\right)^{n}
\end{aligned}
$$

This yields

$$
\begin{aligned}
J_{N} & \leq \sum_{n=N+1}^{\infty}\left(\frac{r}{2^{r}(r-1)}\right)^{n} \\
& =\left(\frac{2^{r}(r-1)}{2^{r}(r-1)-r}\right)\left(\frac{r}{2^{r}(r-1)}\right)^{N+1}
\end{aligned}
$$

whenever $r>r^{*}$. The proof is completed by using the inequality

$$
|\langle\Phi, \psi\rangle| \leq\|\Phi\|_{-\rho,-p, V} \cdot\|\psi\|_{\rho, p, V}
$$

REMARK 3.2 The estimates in the theorem are not optimal. It has not been a goal here to find the best possible bounds.

REMARK 3.3 With obvious modifications, the above theorem can be worked out for smooth random variables $\phi \in(\mathcal{S})^{\rho, p, V}, \rho \in[0,1], p \in \mathbb{N}_{0}$.

EXAMPLE 3.4 Let $p=q+2$. Hence $r=2$. A calculation of the constants give $C_{1}(2)=$ $\frac{1}{2}, C_{2}(2)=2$ and $\frac{r}{2^{r}(r-1)}=1 / 2$. This yields the estimate

$$
\left\|\Phi-\Phi^{N, K}\right\|_{-\rho,-p, V}^{2} \leq \frac{1}{2}\|\Phi\|_{-\rho,-q, V}^{2} \cdot\left[K^{-1}+\left(\frac{1}{2}\right)^{N-1}\right]
$$

If we choose $K=2^{\mathrm{N}-1}$ and define

$$
\Phi^{N}:=\Phi^{N, 2^{N-1}}
$$

we have the estimate

$$
\left\|\Phi-\Phi^{N}\right\|_{-p,-p, V}^{2} \leq\|\Phi\|_{-p,-q, V}^{2} \cdot\left(\frac{1}{2}\right)^{N-1}
$$


EXAMPLE 3.5 Approximation of the Wick product. Fix a constant $\delta>1 / 2$ and let $p=q+2$. For $\Phi, \Psi \in(\mathcal{S})^{-\rho,-q, V}$, we have from a modified version of [HØUZ, proposition 3.3.2] and above example

$$
\begin{aligned}
\left\|\Phi \diamond \Psi-\Phi^{N, K} \diamond \Psi^{N, K}\right\|_{-\rho,-(p+\delta), V}^{2} \leq & 2\left\|\Psi \diamond\left(\Phi-\Phi^{N, K}\right)\right\|_{-\rho,-(p+\delta), V}^{2} \\
& +2\left\|\Phi \diamond\left(\Psi-\Psi^{N, K}\right)\right\|_{-\rho,-(p+\delta), V}^{2} \\
\leq & 2 C\|\Psi\|_{-\rho,-p, V}^{2} \cdot\left\|\Phi-\Phi^{N, K}\right\|_{-p,-p, V}^{2} \\
& +2 C\|\Phi\|_{-\rho,-p, V}^{2}\left\|\Psi-\Psi^{N, K}\right\|_{-p,-p, V}^{2} \\
\leq & 4 C\|\Psi\|_{-\rho,-q, V}^{2} \cdot\|\Phi\|_{-\rho,-q, V}^{2}\left(K^{-1}+\left(\frac{1}{2}\right)^{N+1}\right)
\end{aligned}
$$

where $C$ is a constant independent of $\Phi, \Psi$.

\section{$\S 4$ The stochastic finite element method in $\mathbb{R}^{2}$}

We will now consider the case $V=H_{0}^{1}(D)$ where $D$ is an open, bounded domain in $\mathbb{R}^{2}$ satisfying $\bar{D}=\cup_{i=1}^{Q} \bar{T}_{i}$ for some $Q \in N$ and

1. $T_{i}(1 \leq i \leq Q)$ are open triangles.

2. $T_{i} \cap T_{j}=\emptyset$ for $i \neq j$.

3. For $i \neq j$ the set $\overline{\mathrm{T}}_{i} \cap \overline{\mathrm{T}}_{\mathfrak{j}}$ is either

(a) empty, or

(b) a common side of the elements $T_{i}$ and $T_{j}$, or

(c) a common edge of the elements $T_{i}$ and $T_{j}$.

Note that this implies that $D$ has a polygonal shape.

Let now $V_{M}$ be the $M$-dimensional subspace of $H_{0}^{1}(D)$ defined by the piecewise linear functions

$$
\begin{aligned}
V_{M}:=\left\{u \in C(\bar{D}): u=0 \text { on } \partial D ; \text { on each } T_{i}(1 \leq i \leq Q)\right. \\
\text { the function agrees with a linear function, i.e., } \left.u(x, y)=\gamma_{i 1}+\gamma_{i 2} x+\gamma_{i 3} y \text { on } T_{i}\right\}
\end{aligned}
$$

We will by $u^{M}$ denote the projection of an element $u \in H_{0}^{1}(D)$ onto $V_{M}$.

Let $\Phi \in(\mathcal{S})^{-\rho,-q, 2}$ be given as

$$
\Phi=\sum_{\alpha \in \mathcal{J}} c_{\alpha} H_{\alpha}
$$


and for $\mathrm{N}, \mathrm{K}, \mathrm{M} \in \mathrm{N}$ define the finite element approximation

$$
\Phi^{N, K, M}:=c_{0}^{M}+\sum_{n=1}^{N} \sum_{k=1}^{K} \sum_{\alpha \in \mathcal{A}_{n, k}} c_{\alpha}^{M} H_{\alpha}
$$

THEOREM 4.1 Suppose $h>0$ is the maximum length of the sides of the triangles $\left\{T_{i}\right\}_{i=1}^{Q}$. For any $\psi \in(\mathcal{S})^{p, k, 1}$ there exists a constant $A>0$ such that

$$
\begin{aligned}
& \left|\left\langle\Phi-\Phi^{N, K, M}, \Psi\right\rangle\right| \leq \\
& \quad\left(\sqrt{\left(C_{1}(r) \cdot K^{1-r}+C_{2}(r) \cdot\left(\frac{r}{2^{r}(r-1)}\right)^{N+1}\right.} \cdot\|\Phi\|_{-\rho,-q, 1}+A h\|\Phi\|_{-\rho,-p, 2}\right) \cdot\|\psi\|_{\rho, p, 1}
\end{aligned}
$$

\section{PROOF:}

Since

$$
\begin{aligned}
\Phi-\Phi^{N, K, M} & =\Phi-\Phi^{N, K}+\Phi^{N, K}-\Phi^{N, K, M} \\
& =\Phi-\Phi^{N, K}+c_{0}-c_{0}^{M}+\sum_{n=1}^{N} \sum_{k=1}^{K} \sum_{\alpha \in \mathcal{A}_{n, K}}\left(c_{\alpha}-c_{\alpha}^{M}\right) H_{\alpha}
\end{aligned}
$$

we have

$$
\begin{aligned}
\left\|\Phi-\Phi^{N, K, M}\right\|_{-\rho,-p, 1}^{2}= & \left\|\Phi-\Phi^{N, K}\right\|_{-\rho,-p, 1}^{2} \\
& +\left\|c_{0}-c_{0}^{M}+\sum_{n=1}^{N} \sum_{k=1}^{K} \sum_{\alpha \in \mathcal{A}_{n, k}}\left(c_{\alpha}-c_{\alpha}^{M}\right) H_{\alpha}\right\|_{-\rho,-p, 1}^{2} \\
\leq & \left\|\Phi-\Phi^{N, K}\right\|_{-\rho,-p, 1}^{2}+A^{2} h^{2}\|\Phi\|_{-\rho,-p, 2}^{2}
\end{aligned}
$$

where we have used the inequality $([\mathrm{H}$, Theorem 8.4.4])

$$
\left\|c_{\alpha}-c_{\alpha}^{M}\right\|_{H^{1}(D)} \leq A h\left\|c_{\alpha}\right\|_{H^{2}(D)} \quad(\forall \alpha \in \mathcal{J})
$$

REMARK 4.2 The constant $A$ of theorem 4.1 is dependent on the triangles $\left\{T_{i}\right\}_{i=1}^{Q}$. See $[H$, Page 188] for more information.

\section{$\S 5$ Applications to stochastic partial differential equations}

Suppose that we are given an elliptic bounded bilinear form $a(u, v)$ on $(\mathcal{S})^{-\rho,-q, 1} \times(\mathcal{S})_{0}^{-\rho,-q, 1}$ for some $\rho \in[0,1], q \in \mathbb{N}_{0}$, i.e. there exist constants $C_{a}^{1}, C_{a}^{2}>0$ such that

$$
|a(u, v)| \leq C_{a}^{1}\|u\|_{-\rho,-q, 1} \cdot\|v\|_{-\rho,-q, 1} \text { for } u \in(\mathcal{S})^{-\rho,-q, 1}, v \in(\mathcal{S})_{0}^{-\rho,-q, 1}
$$


and

$$
|a(u, u)| \geq C_{a}^{2}\|u\|_{-p,-q, 1}^{2} \quad \text { for all } u \in(\mathcal{S})_{0}^{-p,-q, 1}
$$

We may now pose the boundary value problem in its variational formulation:

Find $u \in(\mathcal{S})_{0}^{-\rho,-q, 1}$ such that

$$
a(u, v)=(f, v)_{-p,-q, 1}
$$

for all $v \in(\mathcal{S})_{0}^{-\rho,-q, 1}$ where $f \in(\mathcal{S})^{-\rho,-q, 1}$.

The existence and uniqueness of this equations follows from the Lax-Milgram theorem. For numerical purposes we are interested in the stochastic Ritz-Galerkin solution $u_{R G}^{N, K, M}$, i.e.,

Find $u_{R G}^{N, K, M} \in\left(\mathcal{S}_{N, K, M}\right)^{-p,-q, 1}$ such that

$$
a\left(u_{R G}^{N, K, M}, v\right)=(f, v)_{-p,-q, 1}
$$

for all $\nu \in\left(\mathcal{S}_{\mathrm{N}, \mathrm{K}, \mathrm{M}}\right)^{-\rho,-q, 1}$ where $\left(\mathcal{S}_{\mathrm{N}, \mathrm{K}, \mathrm{M}}\right)^{-\rho,-q, 1}$ is the subspace of $(\mathcal{S})^{-\rho,-q, 1}$ consisting of those $\Phi$ such that

$$
\Phi=c_{0}+\sum_{n=1}^{N} \sum_{k=1}^{K} \sum_{\alpha \in \mathcal{A}_{n, k}} c_{\alpha} H_{\alpha} \quad c_{\alpha} \in V^{M}(\forall \alpha \in \mathcal{J})
$$

We have the following numerical approximation theorem:

THEOREM 5.1 Suppose that $u$ is the solution of (2) with $u \in(\mathcal{S})_{0}^{-p,-q, 1} \cap(\mathcal{S})^{-p,-q, 2}$. Then the stochastic Ritz-Galerkin solution $u_{R G}^{N, K, M}$ given by (3) satisfies for all $v \in(\mathcal{S})^{\rho, q, 1}$

$$
\begin{aligned}
& \left|\left\langle u-u_{R G}^{N, K, M}, v\right\rangle\right| \leq \\
& C \cdot\left(\sqrt{\left(C_{1}(r) \cdot K^{1-r}+C_{2}(r) \cdot\left(\frac{r}{2^{r}(r-1)}\right)^{N+1}\right.} \cdot\|u\|_{-\rho,-q, 1}+A h\|u\|_{-\rho,-p, 2}\right) \cdot\|v\|_{\rho, q, 1}
\end{aligned}
$$

for some constant $C>0$.

\section{PROOF:}

This is [H, Theorem 8.2.1] in connection with theorem 4.1.

REMARK 5.2 Note that the stochastic Ritz-Galerkin solution is of the form

$$
u_{R G}^{N, K, M}=\sum_{i=1}^{M}\left(c_{i, 0} b_{i}+\sum_{n=1}^{N} \sum_{k=1}^{K} \sum_{\alpha \in \mathcal{A}_{n, k}} c_{i, \alpha} b_{i} H_{\alpha}\right)
$$

where $\left\{b_{i}\right\}_{i=1}^{M}$ is any basis of $V^{M}$. The coefficients $c=\left\{c_{\alpha, i}\right\}$ are obtained by solving the system of linear equations given by $L c=g$ where $L_{i j \alpha \rho}=a\left(b_{i} H_{\alpha}, b_{j} H_{\rho}\right)$ and $g_{i, \alpha}=f\left(b_{i} H_{\alpha}\right)$. From a numerical point of view the only remaining obstacle is calculating a numerical value of $\left\{\mathrm{H}_{\alpha}\right\}_{\alpha \in \mathcal{J}}$. This will be dealt with in the appendix. 


\section{Appendix: Simulation of $\mathrm{H}_{\alpha}$}

In this appendix, we consider the problem of simulating the random basis functions $\left\{\mathrm{H}_{\alpha}\right\}_{\alpha \in \mathcal{J}}$ and count the number of basis functions required for a specific cutting (i.e. choice of $N$ and $K$ ). The last task is the same as finding the dimension of the finite dimensional subspace where our approximation lives.

From the section with mathematical preliminaries, we defined $H_{\alpha}$ by

$$
H_{\alpha}(\omega):=\prod_{i=1}^{\ell(\alpha)} h_{\alpha_{i}}\left(\left\langle\omega, \xi_{i}\right\rangle\right)
$$

It follows from (1) that $\left\{\left\langle\omega, \xi_{i}\right\rangle\right\}_{i}$ is a family of independent standard Gaussian random variables. Hence, to simulate $H_{\alpha}$, we need to compute the product of a finite set of independent standard Gaussian variables. In order to do this, we must have an algorithm producing normal random numbers and use the formula

$$
h_{n}(x)=\sum_{k=0}^{\left\lfloor\frac{n}{2}\right\rfloor}\left(-\frac{1}{2}\right)^{k} \frac{n !}{k !(n-2 k) !} x^{n-2 k}
$$

to create Hermite polynomials.

To get a feeling of how computer extensive our method will be, we look at the number of basis functions we get for a certain choice of $\mathrm{N}$ and $\mathrm{K}$ :

Denote by $\#^{k, n}$ the number of basis-functions with $\ell(\alpha)=k$ and $|\alpha| \leq n$. For instance, we see that

$$
\#^{2, N}=\sum_{i=1}^{N} \#^{1, i}=\sum_{i=1}^{N} i=\frac{N(N+1)}{2}
$$

since the number of basis-functions with $\ell(\alpha)=1$ and $|\alpha| \leq i$, $\#^{1, i}$, is seen to be $i$;

$$
\{(1),(2),(3), \ldots,(i)\}
$$

In general we have the relation

$$
\#^{k, N}=\sum_{i=1}^{N} \#^{k-1, i}
$$

which for $k \geq 3$ gives

$$
\#^{k, N}=\sum_{i_{1}=1}^{N} \sum_{i_{2}=1}^{i_{1}} \ldots \sum_{i_{k-1}=1}^{i_{k-2}} i_{k-1}
$$

The total number of basis-functions for $\mathrm{N}$ and $\mathrm{K}$ is

$$
\#(K, N)=1+\sum_{k=1}^{K} \#^{k, N}=\left(\begin{array}{c}
N+K \\
N
\end{array}\right)
$$


We add one to the sum on the right hand side since $H_{0}=1$ is part of the basis!

Observe that we need to calculate $K$ independent Gaussian random variables and the coefficients of $\left\{h_{i}\right\}_{i=1}^{N}$ which we get from formula (4).

Example: From the formula above we see that $\#(3,3)=20$ and $\#(4,4)=70$. So, by just increasing the length and the volume of the multi-indices by 1 , we reach the total of 70 basisfunctions, compared to 20 .

Note that we have the relations

$$
\frac{\#(K+1, N)}{\#(K, N)}=\frac{N+K+1}{K+1}
$$

and

$$
\frac{\#(K, N+1)}{\#(K, N)}=\frac{N+K+1}{N+1}
$$

Acknowledgments: It is a pleasure to thank Bjørn Fredrik Nielsen for suggesting the problem and for many interesting and fruitful discussions.

The second author is supported by VISTA, a research cooperation between The Norwegian Academy of Science and Letters and Den Norske Stats oljeselskap A. S. (Statoil).

\section{References}

[C] P. G. Ciarlet: The finite element method for elliptic problems. Studies in mathematics and its applications. J. L. Lions, G. Papanicolaou, R. T. Rockafellar (editors), Vol. 4, (1978).

[GP] A. Germani and M. Piccioni: Semi-discretization of Stochastic Partial Differential Equations on $\mathbb{R}^{\mathrm{d}}$ by a Finite-element Technique. Stochastics 23, (1988).

[HKPS] T. Hida, H. -H. Kuo, J. Potthoff and L. Streit: White Noise - An Infinite Dimensional Calculus. Monograph, Kluwer Academic Publishers. (1993).

[H] W. Hackbusch: Elliptic Differential Equations, Springer Verlag. (1992).

[HLøUZ] H. Holden, T. Lindstrøm. B. Øksendal, J. Ubøe and T.-S. Zhang: The Pressure Equation for Fluid Flow in a Stochastic Medium. Potensial Analysis, 4, (1995), pp. 655-674.

[HøUZ] H. Holden, B. Øksendal, J. Ubøe and T. -S. Zhang: Stochastic Partial Differential Equations - A Modelling, White Noise Functional Approach -. Monograph in preparation. 
[Hu] Y. Hu: Finite Difference Approximations of the Pressure Equation for Fluid Flow in a Stochastic Medium - A Probabilistic Approach - . Preprint, University of Oslo, Norway. (1995).

[KLS] Yu. G. Kondratiev, P. Leukert and L. Streit: Wick Calculus in Gaussian Analysis. Preprint 637/6/94, University of Bielefeld, Germany. (1994).

[KP] P. E. Kloeden and E. Platen: Numerical Solution of Stochastic Differential Equations. Springer Verlag (1992).

[V] G. Våge: Stochastic Differential Equations and Kondratiev Spaces. Ph. D. thesis, University of Trondheim (NTH), Norway. (1995).

[W] J. B. Walsh: Stochastic Partial Differential Equations. In École d'Été de Probabilités de Saint-Flour XIV-1984. Lecture Notes in Mathematics 1180. Springer Verlag. (1986).

[Z] M. Zakai: On the Optimal Filtering of Diffusion Processes. Z. Wahschein. verw. Geb. 11 (1969). 